\title{
The fusion of hard and soft Pomerons: 3-jet diffractive production
}

\author{
V. A. Khoze ${ }^{1,2}$, A. D. Martin ${ }^{1, a}$, M. G. Ryskin ${ }^{1,2}$, A. G. Shuvaev ${ }^{2}$, I. V. Surnin ${ }^{2,3}$ \\ ${ }^{1}$ Institute for Particle Physics Phenomenology, University of Durham, Durham DH1 3LE, UK \\ ${ }^{2}$ Kurchatov National Research Centre, Petersburg Nuclear Physics Institute, Gatchina, St. Petersburg 188300, Russia \\ ${ }^{3}$ St. Petersburg State University, St. Petersburg, Russia
}

Received: 24 May 2019 / Accepted: 8 July 2019 / Published online: 18 July 2019

(C) The Author(s) 2019

\begin{abstract}
We consider the central exclusive production of high $E_{T}$ jets $p p \rightarrow p+(X+$ dijet $)+p$. In particular we study the possible contamination of the purely exclusive signal by semi-exclusive production where no other secondaries are emitted in one hemisphere, between the highest $E_{T}$ dijet and the recoil proton, while in the other hemisphere a third jet, plus possibly additional hadron activity, is allowed, but still separated from the incoming proton by a large rapidity gap. This process arising from the fusion of a hard and a soft Pomeron has not been considered before. It turns out that it gives a negligible contribution. The calculation involves a careful treatment of the QCD colour structure of the amplitudes.
\end{abstract}

\section{Introduction}

Central Exclusive Production (CEP) of high $E_{T}$ jets is of interest for at least two reasons. First, due to its relatively large cross section it plays the role of a 'standard candle' for the calculations of different CEP cross sections [1]; in particular, for the evaluation of the chance to observe new Beyond the Standard Model (BSM) physics in the clean environment provided by CEP kinematics. Next, due to the $J_{z}=0$ selection rule $[2,3]$, the production of quark jets is strongly suppressed by a factor $m_{q}^{2} / E_{T}^{2}$ (where $m_{q}$ is the quark mass). Therefore in the CEP process we observe the gluon jets with a good purity. ${ }^{1}$ Thus we have a 'gluon factory' which provides an excellent possibility to study gluon jets [5].

Exclusive dijet production $p p \rightarrow p+$ dijet $+p$ is shown symbolically in Fig. 1a. Within the perturbative QCD approach the Pomeron may be described at lowest order in $\alpha_{s}$ by the two gluon exchange diagram, and we are led to

\footnotetext{
${ }^{1}$ In principle, away from the exact forward region there is a $\left|J_{z}\right|=2$ contribution, see e.g. [2,3]. However an explicit calculation in [4] shows that the contribution of such a term is very small $\sim 1 \%$.

a e-mail: a.d.martin@durham.ac.uk
}

diagram Fig. 1b. At the lowest $\alpha_{s}$ order the CEP dijet cross section was calculated in [6]. More precise results accounting for the leading logarithmic corrections were obtained in [7] (see also the reviews $[4,8]$ ).

However experimentally it is challenging to observe only two jets, without any additional secondaries. As a rule besides the two high $E_{T}$ jets there are other particles, with smaller transverse momenta, $p_{t}$, and it is not quite clear whether these particles were produced during the jet hadronization or whether they must be considered as an additional relatively low $E_{T}$ jet. Moreover, it is not excluded that some low $p_{t}$ particles were missed by the detector.

For example, in order to select pure CEP dijets in the CDF experiment [9] the ratio, $R_{j j}$, of the dijet mass, $M_{j j}$, to the mass of the whole central system, $M_{X}$ was plotted. Pure CEP events should correspond to a peak at $R_{j j}=M_{j j} / M_{X}=1$. Unfortunately the peak at $R_{j j}=1$ is not well manifested on the top of the background caused by Double Pomeron Exchange (DPE) contribution, ${ }^{2}$ that is inclusive dijet production in Pomeron-Pomeron collisions (sketched in Fig. 1c). The peak looks more like a shoulder in $R_{j j}$ distributions of events with $E_{T}>10 \mathrm{GeV}$ (Fig. 14a of [9]) and only one point which exceeds the background by $1.5 \sigma$ can be seen in Fig. $14 \mathrm{c}$ for $E_{T}>25 \mathrm{GeV}$.

DPE production can be described in terms of diffractive parton distributions (dPDF). The dPDF, that is the distribution of partons inside the Pomeron, was measured at HERA by selecting Deep Inelastic Scattering (DIS) events with a Large Rapidity Gap (LRG) between the incoming proton and the hadron system produced by a heavy photon (or by selecting events with a leading proton carrying away a large momentum fraction $x_{L} \rightarrow 1$ ) $[11,12]$. The cross section of DPE dijet production is given by the convolution of the 'hard' $2 \rightarrow 2$ matrix element squared with the parton distributions originating from the two Pomerons. It was calculated

\footnotetext{
${ }^{2}$ See the discussion in [10].
} 
Fig. 1 Exclusive $(\mathbf{a}, \mathbf{b})$ and semi-exclusive (c) high $E_{T}$ dijet production via

Pomeron-Pomeron fusion; in the case of diagram (b) the represented schematically by the two gluon exchange perturbative QCD Pomeron is

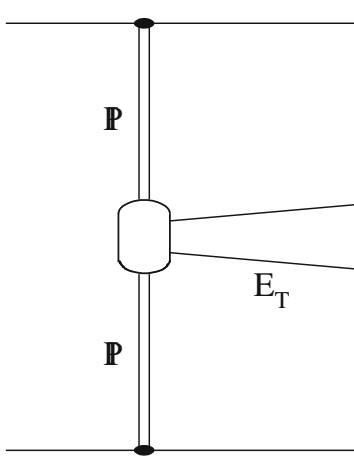

a

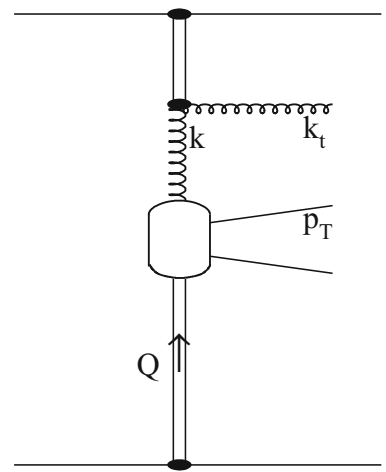

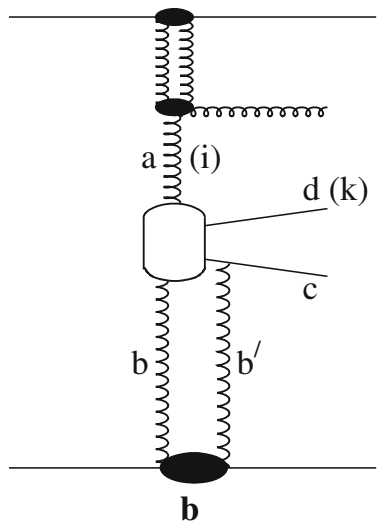

b
Fig. 2 Diagrams for three jet production via the fusion of a 'soft' (upper) and the 'hard' Pomeron. Two highest $p_{T}$ jets are produced in the fusion of a 'hard' Pomeron with the parton (shown as a gluon) from the soft Pomeron PDF. The momenta transferred to the hard matrix element by the hard Pomeron $(Q)$ and the parton from the soft Pomeron $(k)$ together with the momenta of produced jets are indicated in a while the colour indices of the incoming and outgoing gluons (quarks) are shown in $\mathbf{b}$

in $[6,13]$ and implemented in Monte Carlo generators, like e.g. POMWIG [14].

As seen from Fig. 1c, while pure CEP events at the parton level have only two high $E_{T}$ jets, for a DPE process at least four partons/jets are produced - the two high $E_{T}$ jets together with two spectators which are needed to compensate the colour of the parton extracted from the incoming Pomeron; besides this there may be other partons (shown by the dotted lines in Fig. 1c) radiated during the dPDF evolution. For this reason the ratio $R_{j j}<1$ for DPE events.

The aim of this paper is to consider an 'intermediate' configuration between the CEP and DPE possibilities - that is dijet production due to the collison of a parton from the $\mathrm{dPDF}$ of a 'soft' Pomeron on one side with the CEP-like 'hard' Pomeron on the other side. At the lowest $\alpha_{s}$ order this corresponds to three-jet production - a pair of high $E_{T}$ jets and a jet-spectator from the soft Pomeron, see Fig. 2a.

Strictly speaking an analogous three-jet configuration can be produced in pure CEP events as well. One has just to
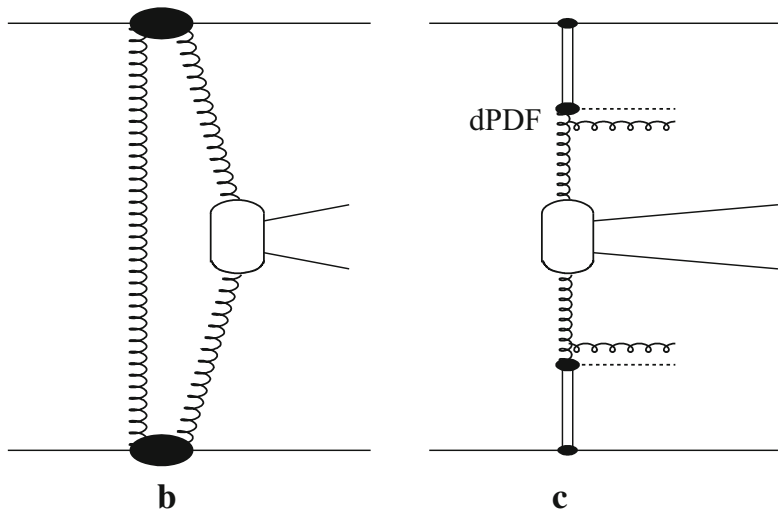

consider the $g g \rightarrow 3$-jet hard subprocess. For a pure CEP case this was done in [15]. Another approach was used in [16]. In this paper the configuration where the third jet has a relatively small momentum jet $\left(k_{t} \ll p_{T}\right)$ was considered. However here it was not the pure exclusive kinematics since the cross section includes the processes in which additional (softer) jets with $k_{t}>1.5 \mathrm{GeV}$ can be radiated.

Note that when the intercept of the Pomeron trajectory, $\alpha_{P}(0)$, is close to 1 there is practically no interference between the pure CEP(3-jet) and the CEP(2-jet) $\otimes D P E$ amplitudes. Soft Pomeron exchange in the DPE amplitude produces an additional (imaginary) factor $i$. Thus we may consider the pure $\mathrm{CEP}(3$-jet $)$ and the $\mathrm{CEP}(2$-jet $) \otimes \mathrm{DPE}$ cross sections separately.

The outline of this paper is as follows. In Sect. 2 we describe the detailed structure of the CEP $\otimes \mathrm{DPE}$ amplitude and show that it can be 'factorized'. That is, the result can be written as the convolution of a hard $2 \rightarrow 2$ matrix element and an integral over much smaller transverse momenta. Next, in Sect. 3, we obtain an expression for the effective luminosity, corresponding to 'hard'-to-'soft' (CEP $\otimes D P E)$ Pomeron-Pomeron fusion, for this semi-exclusive process and to describe its main elements, in particular, the Sudakov $T$-factor and incoming parton distributions. Recall that this effective luminosity includes all the components which are driven by the relatively soft scale fixed by the transverse momentum, $k \ll E_{T}$, of a relatively soft third jet. We discuss different ways to introduce the infrared cutoff, either by an effective gluon mass or by the Sudakov $T$-factor and the incoming unintegrated parton distributions. The hard dijet cross sections of the relevant subprocesses are given in Sect. 4 while the numerical estimates of the expected cross section are presented in Sect. 5. We conclude in Sect. 6.

\section{Soft-hard factorization}

Here by factorization we mean that we can separate the calculation of the hard $2 \rightarrow 2$ subprocess cross section for exclusive production of the high $E_{T}$ dijet from that for semi- 


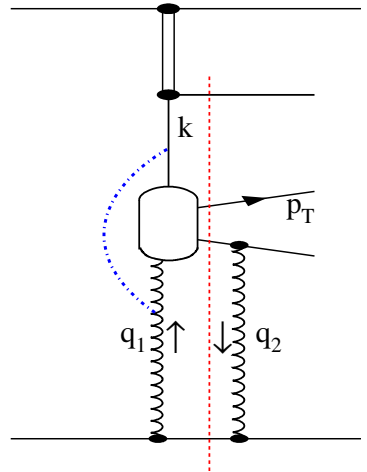

a

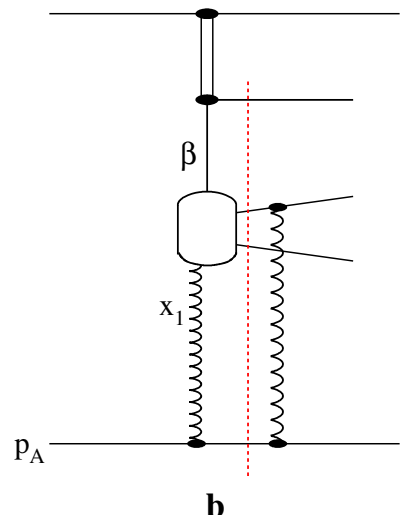

b

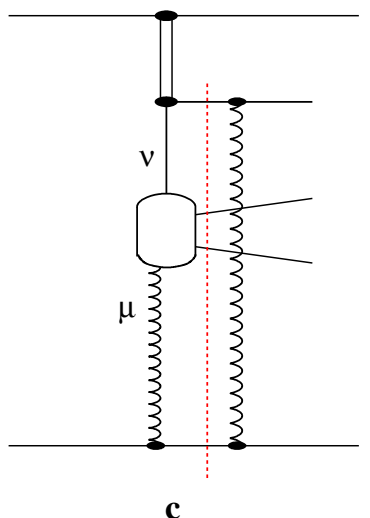

c
Fig. 3 Three diagrams which describe the imaginary part of exclusive 3 -jet production amplitude. The momenta, $q_{1,2}$, of incoming gluons from the hard Pomeron and the parton, $k$, from the soft Pomeron are indicated in $\mathbf{a} ; p_{T}$ is the hard jet momentum. Momentum fractions, $x_{1}$, and $\beta$ carried by the active gluon in hard Pomeron and by the parton in soft Pomeron are shown in $\mathbf{b}$. Lorentz indices of the partons incoming the hard matrix elements are marked in $\mathbf{c}$ exclusive production involving low $k_{t}$ jets. In the calculation of the latter, which includes the incoming parton densities, we introduce an 'effective luminosity', see Sect. 3.3.

This effective luminosity is formed at a rather low scale driven by the relatively small transverse momentum, $k_{t}$, of the third jet. On the other hand the hard dijet cross section occurs at a large $\left(\sim E_{T}\right)$ scale. The Sudakov factor, which accounts for the probability not to radiate additional partons in the interval between $k_{t}$ and $E_{T}$, is included in the 'effective' luminosity.

The amplitude of CEP $\otimes$ DPE dijet production is shown symbolically in Fig. 2a and b where both the soft and hard Pomerons are replaced by two gluon exchange diagrams. Let us denote the transverse momentum ${ }^{3}$ of the parton coming from the upper soft Pomeron by $k$, and the transverse momenta of left and right gluons which compose the bottom Pomeron by $q_{1}, q_{2}$. If $Q$ is the transverse momentum transferred to the recoil proton, then $q_{1,2}=q \pm Q / 2$.

Since the value of $Q^{2}$ is limited by the proton form factor the integrals over $q$ and $k$ (which appear when we calculate the cross section) are ultraviolet (UV) convergent, while the infrared (IR) behaviour is regularized by an effective gluon mass, $m_{g}=0.6-0.8 \mathrm{GeV}$ (see e.g. $[17,18]$ ) or by the proton size (in both cases these reflect the confinement effects) and by the Sudakov $T$-factor which accounts for the probability not to radiate additional relatively soft gluons in the partonPomeron fusion process forming the high $E_{T}$ dijet. This is the same $T$-factor which provides the IR cutoff in a pure CEP amplitude [7,19], see Sect. 3.1.

Since the essential values of $q$ and $k$ are much smaller than the high $E_{T}$ jet transverse momenta, $p_{T}$, we can neglect $q_{1,2}$ and $k$ when calculating the hard matrix element and use

\footnotetext{
3 The transverse momenta are shown in Fig. 3a.
}

the MHV approach (see e.g. [20]) for the hard $2 \rightarrow 2$ subprocess amplitude. Next, recall that in the Pomeron exchange amplitude the imaginary part dominates, while the real part can be restored (if needed) with the help of the well known signature factor. That is we consider only the corresponding imaginary part of the amplitude.

This imaginary part is given by the sum of three diagrams with the cuts shown in Fig. 3 by a vertical dotted line. That is we have to sum three diagrams: two in which the right gluon couples to a high $E_{T}$ jet and the third in which it couples to the upper Pomeron parton-spectator. When the right gluon couples to a high $E_{T}$ jet it does not affect the kinematics shown in Fig. 3a (since $q_{2} \ll p_{T}$ ). On the other hand in Fig. $3 \mathrm{c}$, where the right gluon couples to the spectator, we have to replace the parton momentum $k$ by $k-q_{2}$. Note that the final three jet system is colourless. Therefore the sum of the first two diagrams (Fig. 3a, b) has a colour factor equal (up to the sign) to that of Fig. 3c. ${ }^{4}$ Thus we can select (factorize) the soft part of the amplitude, $I_{\mu \nu}$ which includes the propagators of two (lower) gluons from the hard Pomeron, the propagator of the parton from soft Pomeron and the corresponding polarization vectors. This is the central factor of the 'effective' luminosity which should be further multiplied by the differential hard dijet cross section as it will be explained in Sect. 3.3.

$$
\begin{aligned}
I_{\mu \nu}= & \int d^{2} q \frac{q_{1 \mu}}{\left(q_{1}^{2}+m_{g}^{2}\right)\left(q_{2}^{2}+m_{g}^{2}\right)} \\
& \times\left[\frac{k_{v}}{k^{2}+m_{g}^{2}}-\frac{\left(k-q_{2}\right)_{v}}{\left(k-q_{2}\right)^{2}+m_{g}^{2}}\right] T\left(q, M_{j j}\right) .
\end{aligned}
$$

\footnotetext{
$\overline{{ }^{4} \text { In the } q_{2} \ll k}$ limit, when the gluon wavelength $1 / q_{2}$ is much larger than the size of 3-jet system, the gluon $q_{2}$ probes just the total colour charge of this colourless system and the interaction amplitude vanishes.
} 
We include in (1) the Sudakov factor $T$ which accounts for the absence of radiation of an additional jet since it may strongly affect the infrared behaviour of the integral. This factor is given explicitly in the next subsection. The transverse indices $\mu, v=1,2$ correspond to incoming gluon polarizations which then should be convoluted with the hard matrix element (shown by blob in Fig. 3). In the left part of the amplitude we choose the gluon polarization vectors to be

$\mathbf{e}_{1, \mu} \simeq-\mathbf{q}_{1, \mu} / x_{1} \quad$ and $\quad \mathbf{e}_{k, v} \simeq-\mathbf{k}_{v} / \beta$,

where $x_{1}$ is the lower proton momentum fraction carried by gluon $q_{1}$ while $\beta$ is the upper Pomeron momentum fraction carried by the parton $k^{5}$ This means that we are working in an axial/planar gauge or using Gribov's gauge trick ${ }^{6}$ replacing the (lower) proton 4-momentum, $p_{A, \mu}$, by $-q_{1, \mu} / x_{1}$.

For the second (soft in $x$ ) gluon in the right part of the amplitude of Fig. 3 it is better to use Coulomb polarization, that is to use $g_{\alpha \beta}$ as the spin part of gluon $q_{2}$ propagator. In this case in the upper vertex of the gluon $q_{2}$ we have just $p_{A, \alpha}$. This simplifies the calculation of imaginary part of the diagram, that is the 'cut' between the 'soft'(right) and the 'hard' (left) gluons; recall that the hard matrix element is to the left of the 'cut'. Clearly the integral (1) has no UV divergency. ${ }^{7}$

The values of $I_{\mu \nu}=I_{\mu \nu}(Q, k)$ can be calculated numerically. In order to perform the convolution with the hard matrix element calculated in terms of helicity amplitudes we consider three possibilities: $I^{(0)}, I^{(2)}$ and $I^{(q)}$ corresponding to whether the high $E_{T}$ dijet is produced by gluon-gluon fusion in either the $J_{z}=0$ or $J_{z}=2$ helicity states or by quark-gluon fusion dijet production $q g \rightarrow q g$. We introduce the upper index $I^{(s)}$ (with $s=0,2, q$ ) in order to consider in turn the convolution of the soft part with the different hard matrix elements which may describe either the gluon ( $g g \rightarrow$ dijet) production in $J_{z}=0$ or $\left|J_{z}\right|=2$ helicity states or $q g \rightarrow q g$ production. If the parton $k$ is a quark then the square bracket in (1) should be multiplied by the Dirac matrix $\gamma_{\nu}$. Note that for $g g \rightarrow g g$ dijet production, $J_{z}$ is the projection of the spin of the dijet system on the longitudinal (beam) axis, that is $J_{z}$ corresponds to the difference of helicities of the incoming gluons with momenta $q_{1}$ and $k$.

Thus we calculate

$$
I^{(0)}(k)=\frac{1}{4} \int d^{2} Q\left(I_{x x}+I_{y y}\right)^{2},
$$

\footnotetext{
${ }^{5}$ Factors $1 / x_{1}$ and $1 / \beta$ are not included in $I_{\mu \nu}$ but we will account for them in Sect. 3.3 when calculating the effective luminosity (11).

${ }^{6}$ Recall that the left (with respect to the cut) part of the diagram is gauge invariant.

7 The IR contribution is smeared by the effective gluon mass $m_{g}$ and the $T$-factor.
}

$$
\begin{aligned}
I^{(2)}(k)=\frac{1}{4} \int d^{2} Q\left(\left(I_{x x}+I_{y y}\right)^{2}-4 I_{x x} I_{y y}\right. & \\
& \left.+2 I_{x y} I_{y x}+I_{x y} I_{x y}+I_{y x} I_{y x}\right), \\
I^{(q)}(k)= & \frac{1}{4} \int d^{2} Q\left(I_{x x} I_{x x}+I_{y y} I_{y y}+I_{x y} I_{x y}+I_{y x} I_{y x}\right),
\end{aligned}
$$

where we have used the 'projectors' $\delta_{\mu \nu} \delta_{\mu^{\prime} v^{\prime}}$ for the $J_{z}=$ 0 state and $\left(\delta_{\mu v^{\prime}} \delta_{\nu \mu^{\prime}}+\delta_{\mu \mu^{\prime}} \delta_{\nu v^{\prime}}-\delta_{\mu \nu} \delta_{\mu^{\prime} v^{\prime}}\right)$ for the $J_{z}=$ 2 state. Here the indices $\mu^{\prime}, v^{\prime}$ belong to the complexconjugated amplitude. For quark-gluon fusion the projector is $\delta_{\mu \mu^{\prime}} \delta_{v v^{\prime}}$.

Indeed, the projector $\delta_{\mu \nu}$ means that we select the $J_{z}=0$ state and we have to sum over all possible polarizations of the transverse gluon. That is we obtain $\left(I_{x x}+I_{y y}\right)$. Doing the same with the complex-conjugated amplitude we obtain (3). It is not so strightforward with the $J_{z}=2$ amplitude but multiplying by $\delta_{\mu \nu}$ one can easily check that the product $\left(\delta_{\mu \nu^{\prime}} \delta_{\nu \mu^{\prime}}+\delta_{\mu \mu^{\prime}} \delta_{\nu v^{\prime}}-\delta_{\mu \nu} \delta_{\mu^{\prime} v^{\prime}}\right) \cdot \delta_{\mu \nu}=0$, that is this projector does not contain $J_{z}=0$ state while the remaining states of two gluon system have $\left|J_{z}\right|=2$. Finally in the quark case we just sum up all possible combinations of polarizations.

\section{Soft components of production amplitude}

Here we discuss the various components that are required in the calculation of the cross section for semi-exclusive soft production.

\subsection{The Sudakov $T$ factor}

The Sudakov form factor correction originates from diagrams like that show by the dash-dotted line in Fig. 3a. It accounts for the fact that for exclusive events we do not allow for standard bremsstrahlung from the colour-charged incoming gluon (or quark). Within the leading double logarithmic (DL) approximation the Sudakov $T$-factor reads

$T(q, \mu)=\exp \left(-\frac{N_{c} \alpha_{s}}{4 \pi} \ln ^{2}\left(\mu^{2} / q^{2}\right)\right)$

for the case when parton $k$ is a gluon. When parton $k$ is a quark the colour coefficient $N_{c}=3$ (for the QCD $S U_{c}(3)$ group) must be replaced by $\left(N_{c}+C_{F}\right) / 2=13 / 6$. Accounting for the one-loop running QCD coupling $\alpha_{s}\left(q^{2}\right)=$ $\left(4 \pi / b_{0}\right) / \ln \left(q^{2} / \Lambda_{Q C D}^{2}\right)$, expression (6) takes the form

$$
T_{g}(q, \mu)=\exp \left(\int_{q^{2}}^{\mu^{2}} \frac{d \kappa^{2}}{\kappa^{2}} \frac{N_{c} \alpha_{s}\left(\kappa^{2}\right)}{\pi} \ln (\kappa /(\kappa+\mu))\right) .
$$


In practice we use more precise expressions for the quark and gluon $T$-factors. These can be found in [21].

The upper limit $\mu$ of the integral is taken to be $M_{j j}$, the mass of the high $E_{T}$ dijet system (see [22]), while the lower cutoff, $q^{2}$, reflects the cancellation between the coherent radiation from the $q_{1}$ and $q_{2}$ gluons for the emission of an extra gluon with wavelength larger than the size of the colourless $q_{1}$ and $q_{2}$ gluon pair.

Recall that strictly speaking the full $T$ factor correction depends on a particular jet searching algorithm. Depending on the algorithm some part of bremsstrahlung emission may be allowed and included in high $E_{T}$ jet hadronization.

\subsection{Incoming parton distributions}

Note that, due to the cancellation for $k \gg q$ between the first and the second terms in the square brackets of (1), the relevant values of momentum $k$ is of the order of $q$. The integral is UV convergent. The dominant contribution comes from the low $q, k$ domain with an IR cutoff provided by the $T$-factor (6) or (for the case of not too large $E_{T}$, when the $T$-factor is ineffective) by confinement, or by an effective gluon mass $m_{g}$.

Note that the calculation of the diagrams of Fig. 3 require knowledge of unintegrated parton distributions, $f^{a}$. For the 'soft' (upper) Pomeron these explicitly depend on the transverse momentum $k$, that is $f^{a}=f^{a}\left(x_{I P}, \beta, k, \mu\right.$; $\left.t\right)$ with $a=g, q$, where the arguments are defined below. These unintegrated distributions can be obtained from the (integrated) dPDFs with the help of the KMR/MRW prescriptions $[21,23]$. Actually here we use the simplified form

$$
f^{a}\left(x_{I P}, \beta, k, \mu ; t\right)=\frac{\partial}{\partial \ln k^{2}}\left[T_{a}(k, \mu) x a^{D}\left(x_{I P}, \beta, k^{2} ; t\right)\right],
$$

where $a^{D}$ is the integrated diffractive parton distribution (dPDF) and $x=x_{I P} \beta$. For $a^{D}$ we take the H1 fit B parametrization which satisfactorily describes the diffractive DIS data [11]. Note that the corresponding dPDF depends on three arguments - the proton momentum fraction, $x_{I P}$, carried by the Pomeron, the Pomeron momentum fraction, $\beta$, carried by the parton, and the scale $\mu$. Besides this there is the dependence of the Pomeron flux on the momentum transferred, $t$ (see the parametrization presented in [11]).

The situation for the 'hard' (bottom) Pomeron is different. When the scale is relatively large, say $q_{1,2}>1-2 \mathrm{GeV}$, we have to replace the two gluon exchange diagram (i.e. the Low-Nussinov two gluon Pomeron [24,25]) by the Generalized Parton Distribution (GPD) function ${ }^{8}$ of proton. That is instead of the usual (unskewed) PDF we deal with the GPD

\footnotetext{
${ }^{8}$ See e.g. [26] for review.
}

since the momentum transferred through our Pomeron is not zero. In particular, the momentum fraction $x_{2} \ll x_{1}$. Since we are looking for LRG events, the value of $x_{1} \ll 1$ is itself small and so the Generalized function GPD can be obtained from the known usual PDF $[27,28]$. In the limit $x_{2} \ll x_{1}$ the ratio $R_{g}=\mathrm{GPD} / \mathrm{PDF}$ is given by

$R_{g}=\frac{2^{2 \lambda+3}}{\sqrt{\pi}} \frac{\Gamma(\lambda+5 / 2)}{\Gamma(\lambda+4)}$,

when the small $x$ behaviour of gluons is described by $x g(x) \propto x^{-\lambda}$ and where $\Gamma$ is the Gamma function.

\subsection{Effective luminosity}

As shown above, the three jet cross section caused by the fusion of a 'soft' and a 'hard' Pomeron can be written as the convolution of the effective luminosity with the cross section of the hard subprocess. The non-trivial point is that now the third jet (with the smaller transverse momentum $k$ distribution) is included in an effective 'luminosity factor', $L\left(x_{1}, \beta, x_{I P} ; k, M_{j j}\right)$. That is the final cross section reads

$\frac{M_{j j}^{2} d \sigma}{d Y_{j j} d M_{j j}^{2} d \beta d k^{2}}=L\left(x_{1}, \beta, x_{I P} ; k, M_{j j}\right) \frac{d \hat{\sigma}}{d \hat{t}}$,

where $Y_{j j}$ is the rapidity of the high $E_{T}$ dijet system and $x_{I P}$ is the fraction of the proton's momentum carried by the soft (upper) Pomeron.

Using the notation $I^{(s)}$ of Eqs. (3-5) we can write the effective luminosity as

$L^{(s)}\left(x_{1}, \beta, x_{I P} ; k, M_{j j}\right)=\frac{\pi \alpha_{s}\left(k^{2}\right)}{4} I^{(s)}(k) f^{a}\left(x_{I P}, \beta, k, \mu\right) S^{2}$.

Here $f^{a}(a=g, q)$ denotes the unintegrated diffractive parton distribution produced by the soft (upper) Pomeron; and $\beta$ is the fraction of the soft Pomeron's momentum carried by the parton $k$. The factor $S^{2}$ is the soft gap survival factor which accounts for the absorptive corrections (see, for example, the review in [29]). In other words $S^{2}$ is the probability that the LRG will not be filled by secondaries produced by additional inelastic interactions which may accompany the main process of Fig. 3.

Note that by using integral (1) in (11) we have assumed that the hard (lower) Pomeron is described just by the twogluon exchange diagram. To be more precise the two-gluon exchange factor $1 /\left(\left(q_{1}^{2}+m_{g}^{2}\right)\left(q_{2}^{2}+m_{g}^{2}\right)\right)$ should be replaced by the unintegrated GPD function. That is, when calculating the $I^{(s)}(k)$ of Eqs. (3-5) we have to use 


$$
\begin{aligned}
I_{\mu \nu}=\int & d^{2} q \frac{q_{1 \mu} \cdot F_{g}\left(x_{1}, x_{2} ; q, \mu\right)}{\left(q_{1}^{2}+m_{g}^{2}\right)\left(q_{2}^{2}+m_{g}^{2}\right)} \\
& \times\left[\frac{k_{t, v}}{k^{2}+m_{g}^{2}}-\frac{\left(k-q_{2}\right)_{t, v}}{\left(k-q_{2}\right)^{2}+m_{g}^{2}}\right] .
\end{aligned}
$$

The GPD function $F_{g}$ in our $x_{2} \ll x_{1}$ limit can be written in the simplified form $[5,30]$

$$
F_{g}\left(x_{1}, x_{2} ; q, \mu\right)=R_{g} \frac{\partial}{\partial \ln q^{2}}\left[\sqrt{T_{g}(q, \mu)} x_{1} g\left(x_{1}, q^{2}\right)\right],
$$

where $g\left(x_{1}, q^{2}\right)$ is the usual integrated gluon distribution and $R_{g}$ is given by (9). Here and below we put for simplicity $Q=$ 0 and omit this argument from now on. Note that working in terms of unintegrated distributions we have no explicit $T$ factor in (12). It is already included in the expressions for $f^{a}$ and $F_{g}$ (see (13)).

At first sight it looks as if the integral (12) over $q^{2}$ has a logarithmic $d q^{2} / q^{2}$ form for $q^{2} \ll k^{2}$, and instead of the unintegrated distribution (13) one can use the full GPD function taken at a scale equal to $k^{2}$. However, this is not true. Expanding the expression in the square brackets in (12) over the $q / k$ ratio and averaging over the azimuthal angle, we see that the first term proportional to $q / k$ vanishes, while the remaining terms do not have a logarithmic structure. Simultaneously the integral over the lowest jet momentum $k$ also has a non-logarithmic form.

Note also that in the denominators $1 / k^{2}$ and $1 /\left(k-q_{2}\right)^{2}$, corresponding to a parton radiated from the soft Pomeron in (12), we have to keep the full parton virtuality $k^{2}=k_{t}^{2} /(1-$ $\beta$ ). Therefore, with the previous notation $k^{2}=k_{t}^{2}$ we must replace (12) by

$$
\begin{aligned}
I_{\mu v}= & \int d^{2} q \frac{q_{1 \mu} \cdot F_{g}\left(x_{1}, x_{2} ; q, \mu\right)}{\left(q_{1}^{2}+m_{g}^{2}\right)\left(q_{2}^{2}+m_{g}^{2}\right)} \\
& \times\left[\frac{(1-\beta) k_{v}}{k^{2}+q_{I P}^{2}}-\frac{(1-\beta)\left(k-q_{2}\right)_{v}}{\left(k-q_{2}\right)^{2}+q_{I P}^{2}}\right] .
\end{aligned}
$$

Finally for the unintegrated soft Pomeron distribution $f^{a}$ we have taken (8) from the fit $B$ parametrization of the H1 collaboration [11] assuming that at $Q_{k}^{2}<Q_{0}^{2}$ the values of $a^{D}\left(x_{I P}, \beta, Q_{k} ; t\right)$ and $\partial a^{D}\left(x_{I P}, \beta, Q_{k} ; t\right) / \partial \ln Q_{k}^{2}$ are frozen; that is, equal to their value at $Q=Q_{0}$. For very small $Q_{k}<1 \mathrm{GeV}$ we put $a^{D}(\ldots) \propto Q_{k}^{2}$ but this negligibly changes the results in comparison with the simple 'frozen' assumption (recall that here $Q_{k}^{2}=k_{t}^{2} /(1-\beta)$ ). Note that in (14) an effective infrared cutoff $q_{I P}$, corresponding to the soft Pomeron size, is included in order to have the possibility of considering Pomerons with a size smaller than that given by the cutoff $m_{g}$; we put $q_{I P}=1 \mathrm{GeV}$ (or $0.2 \mathrm{GeV}$ ).

\section{Hard dijet $(2 \rightarrow 2)$ cross section}

The cross section of hard subprocess of dijet production is calculated using the MHV formalism [20]. The only nontrivial point is that now we are not looking for the usual colour-averaged cross sections, but for cross sections with the high $E_{T}$ dijet in either a colour-octet state (if the parton $k$ is a gluon) or a colour-triplet state (if it is a quark). That is, the hard matrix element $\mathcal{M}$ for the differential cross section

$$
\frac{d \hat{\sigma}}{d t}=\frac{|\mathcal{M}|^{2}}{16 \pi \hat{s}^{2}}
$$

is given as follows:

$$
\begin{aligned}
& \mathcal{M}_{\lambda_{c} \lambda_{d}}^{a c d}\left(g g \rightarrow g g ; J_{z}=0\right) \\
& =\frac{1}{4\left(N_{c}^{2}-1\right)} \sum_{b, e} f^{a b e} \sum_{\lambda_{e}, \lambda_{b}} \delta_{\lambda_{b} \lambda_{e}} M_{\lambda_{e} \lambda_{b} \lambda_{c} \lambda_{d}}^{e b c d}, \\
& \mathcal{M}_{\lambda_{c} \lambda_{d}}^{a c d}\left(g g \rightarrow g g ; J_{z}=2\right) \\
& =\frac{1}{4\left(N_{c}^{2}-1\right)} \sum_{b, e} f^{a b e} \sum_{\lambda_{e}, \lambda_{b}} \delta_{\lambda_{b}-\lambda_{e}} M_{\lambda_{e} \lambda_{b} \lambda_{c} \lambda_{d}}^{e b c d}, \\
& \mathcal{M}_{\lambda_{c} \lambda_{d}}^{a i k}\left(g g \rightarrow q \bar{q} ; J_{z}=0\right) \\
& =\frac{1}{4\left(N_{c}^{2}-1\right)} \sum_{b, e} f^{a b e} \sum_{\lambda_{e}, \lambda_{b}} \delta_{\lambda_{b} \lambda_{e}} M_{\lambda_{e} \lambda_{b} \lambda_{i} \lambda_{k}}^{e b i k}, \\
& \mathcal{M}_{\lambda_{c} \lambda_{d}}^{a i k}\left(g g \rightarrow q \bar{q} ; J_{z}=2\right) \\
& =\frac{1}{4\left(N_{c}^{2}-1\right)} \sum_{b, e} f^{a b e} \sum_{\lambda_{e}, \lambda_{b}} \delta_{\lambda_{b}-\lambda_{e}} M_{\lambda_{e} \lambda_{b} \lambda_{i} \lambda_{k}}^{e b i k},
\end{aligned}
$$

and

$$
\begin{aligned}
& \mathcal{M}_{\lambda_{i} \lambda_{b} \lambda_{c} \lambda_{k}}^{i c k}(g q \rightarrow g q) \\
& =\frac{1}{2\left(N_{c}^{2}-1\right)} \sum_{b, i^{\prime}} t_{i i^{\prime}}^{b} \sum_{\lambda_{i^{\prime}}} \delta_{\lambda_{i} \lambda_{i^{\prime}}} M_{\lambda_{i^{\prime}} \lambda_{b} \lambda_{c} \lambda_{k}}^{i^{\prime} b c k}
\end{aligned}
$$

where $a, b, c, d, e=1,2, \ldots, 8\left(i, i^{\prime}, k=1,2,3\right)$ are the gluon (quark) colour indices, while the $\lambda_{a}, \lambda_{b}, \ldots= \pm 1$ are the helicities of the corresponding gluon or quark $\left(\lambda_{i}=\right.$ $\pm 1 / 2) . M_{\lambda_{e} \lambda_{b} \lambda_{c} \lambda_{d}}^{e b c d}(g g \rightarrow g g)$ and $M_{\lambda_{i^{\prime}} \lambda_{b} \lambda_{c} \lambda_{k}}^{i c k}(q g \rightarrow q g)$ are the conventional matrix elements. These formulae, with the unusual clour strcture exposed, are needed for the calculation of three (or more) jet production.

With this unusual colour structure we now find that the hard cross sections, averaged over the colours and helicities of incoming partons and summed for the outgoing partons, read:

$$
\begin{aligned}
& \frac{d \hat{\sigma}^{(0)}(g g \rightarrow g g)}{d t}=\frac{\pi \alpha_{s}^{2} N_{c}^{3}}{\left(N_{c}^{2}-1\right)^{2}} \frac{1}{p_{T}^{4}}\left(1-\frac{4 p_{T}^{2}}{\hat{s}}\right), \\
& \frac{d \hat{\sigma}^{(0)}(g g \rightarrow q \bar{q})}{d t}=0,
\end{aligned}
$$




$$
\begin{aligned}
& \frac{d \hat{\sigma}^{(2)}(g g \rightarrow g g)}{d t}=\frac{\pi \alpha_{s}^{2} N_{c}^{3}}{\left(N_{c}^{2}-1\right)^{2}} \\
& \quad \times \frac{1}{p_{T}^{4}}\left(1-\frac{4 p_{T}^{2}}{\hat{s}}\right)\left(1-4 p_{T}^{2} / \hat{s}+4 p_{T}^{4} / \hat{s}^{2}\right) \\
& \frac{d \hat{\sigma}^{(2)}(g g \rightarrow q \bar{q})}{d t} \\
& =\frac{\pi \alpha_{s}^{2} N_{c}^{2}}{4\left(N_{c}^{2}-1\right)^{2}} \frac{1}{p_{T}^{2} \hat{s}}\left(1-\frac{4 p_{T}^{2}}{\hat{s}}\right)\left(1-2 p_{T}^{2} / \hat{s}\right) \\
& \frac{d \hat{\sigma}^{q}(g q \rightarrow g q)}{d t}=\frac{\pi \alpha_{s}^{2}}{N_{c}^{3}\left(N_{c}^{2}-1\right)}\left(\frac{2 N_{c}^{2} \hat{u}+\hat{t}}{\hat{t}}\right)^{2} \frac{\hat{u}^{2}+\hat{s}^{2}}{16 \hat{s} \hat{u}} .
\end{aligned}
$$

where

$\hat{s}=M_{j j}^{2}, \hat{t}=\hat{s}(1-\cos (\theta)) / 2, \hat{u}=\hat{s}(1+\cos (\theta)) / 2$,

are the Mandelstam variables corresponding to the hard subprocess; $\theta$ is the scattering angle in dijet rest system; and $p_{T}^{2}=\hat{t} \hat{u} / \hat{s}$.

Note that in the case of gluon-gluon collisions the factor $\left(1-4 p_{T}^{2} / \hat{s}\right)$ vanishes at $\theta=\pi / 2$. This reflects the fact that we deal with a $g g$ system in the asymmetric ( $f^{a b c}$ tensor) colour-octet state. Therefore the corresponding (symmetric) $g g$ wave function has a zero at $90^{\circ}$.

\section{Numerical example}

The above formulation allows the evaluation of the role of 'soft-hard' Pomeron fusion as a background to CEP high $E_{T}$ jet production. As a numerical example we calculate the cross section of central semi-exclusive dijet production at $\sqrt{s}=13 \mathrm{TeV}$ for jets with $p_{T}=30 \mathrm{GeV}$ and rapidity of dijet system $Y_{j j}=0$. We take the dijet scattering angle $\theta=45^{\circ}$ (in dijet c.m.s.) in order not to affect the result by the vanishing of gluon-gluon induced colour-octet cross sections $(21,23,24)$ at $\theta=90^{\circ}$. That is the two high $E_{T}$ jets are separated by the pseudorapidity interval $\Delta \eta=3.5$ (corresponding to jets with $\eta_{j}= \pm 1.75$ ). We sum over all types of parton jets. That is, a jet may be a gluon or a light quark $(u, d, s, c)$ jet. ${ }^{9}$ Next the dijet system is accompanied by a softer third jet, allowing transverse momentum $k_{3}<p_{T}$. Specifically we consider $k_{3}<3,6$ and $10 \mathrm{GeV}$. In addition we allow radiation from the soft Pomeron with $k_{i}<k_{3}$. The results are shown in Fig. 4; we plot the distribution over the ratio $M_{j j} / M_{X}=R_{j j}=\beta$ and compare this with the cross section of pure exclusive dijet production (shown by the dashed line in the upper right corner). For the 'hard' Pomeron

\footnotetext{
${ }^{9}$ We take the survival factor $S^{2}=0.02$ as in model-2 of [31].
}

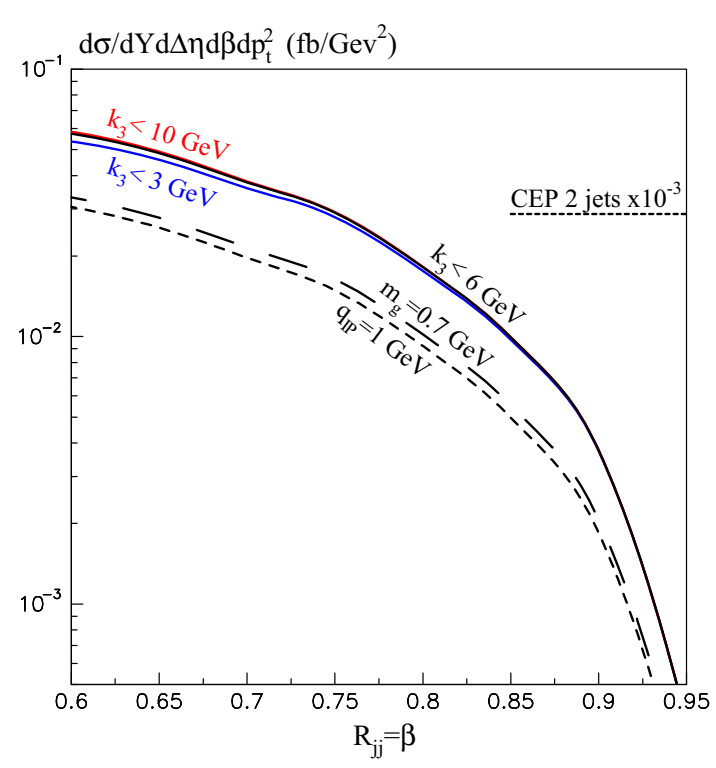

Fig. 4 The cross section of three jet semi-exclusive central production integrated over the third jet transverse momentum $k_{3}$ up to 3, 6 and 10 $\mathrm{GeV}$ (respectively shown by blue, black and red continuous curves) at $\sqrt{s}=13 \mathrm{TeV}$ as the function of the ratio $R_{j j}=M_{j j} / M_{X}$. The two high $E_{T}$ jets have $p_{T}=30 \mathrm{GeV}$ and pseudorapidities $\eta_{j}= \pm 1.75$, $Y_{j j}=0$. An infrared cutoff $m_{g}=q_{I P}=0.2 \mathrm{GeV}$ was used for the main calculation shown by continuous curves. Two alternative choices of cutoff are also shown: $m_{g}=0.7 \mathrm{GeV}$ corresponding to the long dashed curve with $k_{3}<6 \mathrm{GeV}$, while $q_{I P}=1 \mathrm{GeV}$ and $m_{g}=0.2$ $\mathrm{GeV}$ corresponds to the lower dashed curve $\left(k_{3}<6 \mathrm{GeV}\right)$. The pure exclusive dijet cross section reduced by factor of 1000 is shown by the horizontal short dashed line. We used the integrated MMHT2014 parton distributions [32] (for the 'hard' Pomeron) and the H1 fit B for the diffractive parton distributions [11] for the soft Pomeron

we use the integrated parton distributions of [32] and for the 'soft' Pomeron) the diffractive parton distributions of $\mathrm{H} 1 \mathrm{fit}$ $\mathrm{B}[11] .^{10}$

As emphasized above, the major contribution comes from the relatively low transverse momenta of the third jet; the difference between the blue $\left(k_{3}<3 \mathrm{GeV}\right)$ and the red $\left(k_{3}<10\right.$ $\mathrm{GeV})$ curves is rather small. This fact justifies the possibility of calculating such a 3 -jet cross section using the factorization approach.

All the continuous curves were calculated using a weak infrared cutoff of about $1 \mathrm{fm}\left(m_{g}=q_{I P}=0.2 \mathrm{GeV}\right)$. A stronger IR cutoff, shown by the dashed curves, reduces the cross section by a factor of about two.

In comparison with the pure exclusive (CEP) dijet production (shown by the horizontal dotted line) the contribution of production driven by the soft-hard Pomeron fusion mechanism is practically negligible. It is smaller by three orders of magnitude. Recall that the probability of radiation of a third jet from the hard matrix element in hard-hard Pomeron

\footnotetext{
10 The NLO DGLAP evolution of fit B input distributions was performed by QCDNUM program [33].
} 
(CEP) fusion is about $10 \%$ [15]. The role of DPE dijet production was studied in [16]. There the authors accounted for the fact that due to detector resolution and the jet searching algorithm, the CEP peak is washed out and has a maximum at $R_{j j} \simeq 0$.9. Allowing for the initial-state shower, that is including the DPE contribution, the cross section obtained in [16] already at $R_{j j}=0.9$ exceeds the CEP component by more than a factor of 2 .

Such a strong suppression of 'hard-soft' Pomeron fusion is caused by the asymmetric structure of the amplitude. Besides the $\alpha_{s}$ suppression (in which the small value of coupling is not compensated by the logarithmic transverse momentum integration $\int d k_{t}^{2} / k_{t}^{2}$ ) our hard matrix element has an additional smallness due to the fact that we are looking for the production of large $E_{T}$ jets in an asymmetric colour octet state. Recall that the elementary dijet cross sections (21-24) vanish at $\theta=90^{\circ}$. Thus the absence of large logarithms in the $k_{t}$ and $q_{t}$ integrals over the incoming parton momenta and the numerically small factors coming from the asymmetric angular integrations, result in a very small contribution of this mechanism to the final high $E_{T}$ jets cross section.

\section{Conclusion}

We have considered the possibility of semi-exclusive high $E_{T}$ dijet production accompanied by a third jet with smaller transverse momentum plus the possibility of additional radiation coming from soft Pomeron spectators. That is jet production from soft-hard Pomeron fusion.

We have shown that the cross section of such a process can be calculated using the factorization of hard dijet cross sections and an effective luminosity which describes the probability to find appropriate incoming partons and to emit the third jet. Moreover the role of the infrared cutoff was studied.

We found that the contribution of this channel is quite small in comparison with pure CEP dijet production. This fact, which was not evident a priori, greatly simplifies the calculation and interpretation of the exclusive (and semiexclusive) high $E_{T}$ jet production since one can neglect the hard-soft Pomeron fusion contribution.

Note that the cross section that we have calculated is just the simplest example of processes which may arise from the fusion of two different structures of the Pomeron. The result that processes caused by soft-hard Pomeron fusion can be factorized, as the convolution of a pure hard matrix element and an effective luminosity calculated at a much lower scale, has a universal nature. That is, the effective luminosity can be applied to other central diffractive processes. However, as we have shown in our 3-jet example, the cross section arising from hard-soft Pomeron fusion turns out to be small. Of course, in cases where the original CEP amplitude is suppressed, for example by the $J_{z}=0$ selection rule as in $b \bar{b}$ production, then the hard-soft fusion contribution may be noticeable.

Acknowledgements MGR thanks the IPPP at the University of Durham for hospitality. VAK acknowledges support from a Royal Society of Edinburgh Auber award.

Data Availibility Statement This manuscript has no associated data or the data will not be deposited. [Authors' comment: No data deposited as this is a theory project.]

Open Access This article is distributed under the terms of the Creative Commons Attribution 4.0 International License (http://creativecomm ons.org/licenses/by/4.0/), which permits unrestricted use, distribution, and reproduction in any medium, provided you give appropriate credit to the original author(s) and the source, provide a link to the Creative Commons license, and indicate if changes were made. Funded by $\mathrm{SCOAP}^{3}$.

\section{References}

1. L.A. Harland-Lang, V.A. Khoze, M.G. Ryskin, W.J. Stirling, Eur. Phys. J. C 69, 179-199 (2010)

2. V.A. Khoze, A.D. Martin, M.G. Ryskin, Eur. Phys. J. C 19, $477-$ 483 (2001). (Erratum: Eur. Phys. J. C20(2001), 599 )

3. V.A. Khoze, A.D. Martin, M.G. Ryskin, arXiv:hep-ph/0006005 (2000)

4. L.A. Harland-Lang, V.A. Khoze, M.G. Ryskin, W.J. Stirling, Int. J. Mod. Phys. A 29, 1430031 (2014)

5. V.A. Khoze, A.D. Martin, M.G. Ryskin, Eur. Phys. J. C 23, 311 (2002)

6. A. Berera, J.C. Collins, Nucl. Phys. B 474, 183 (1996)

7. V.A. Khoze, A.D. Martin, M.G. Ryskin, Phys. Rev. D 56, 5867 (1997)

8. M.G. Albrow, T.D. Coughlin, J.R. Forshaw, Prog. Part. Nucl. Phys. 65, $149(2010)$

9. CDF Collaboration (T. Aaltonen et al.), Phys. Rev. D 77 (2008) 052004

10. V.A. Khoze, A.D. Martin, M.G. Ryskin, Eur. Phys. J. C 48, 467 (2006)

11. H1 Collaboration (A. Aktas et al.) Eur. Phys. J. C 48 (2006) 715748, H1 Collaboration (A. Aktas et al.) Eur. Phys. J. C 48, 749-766 (2006)

12. ZEUS Collaboration (S. Chekanov et al.). Eur. Phys. J. C 38, 43-67 (2004)

13. M. Boonekamp, R.B. Peschanski, C. Royon, Phys. Rev. Lett. 87, 251806 (2001)

14. B.E. Cox, J.R. Forshaw, Compute. Phys. Commun. 144, 104 (2002)

15. L.A. Harland-Lang, V.A. Khoze, M.G. Ryskin, Eur. Phys. J. C 76, 9 (2016) (Sect. 4.1)

16. L. Lonnblad, R. Zlebcík, Eur. Phys. J. C 76, 668 (2016)

17. G. Parisi, R. Petronzio, Phys. Lett. B 94, 51-53 (1980). ( $m_{g}=0.8$ $\mathrm{GeV}$ )

18. M. Consoli, J.H. Field, Phys. Rev. D 49, 1293-1301 (1994). ( $m_{g}=$ $0.66 \pm 0.08 \mathrm{GeV})$

19. V.A. Khoze, A.D. Martin, M.G. Ryskin, Phys. Lett. B 401, 330 (1997)

20. M.L. Mangano, S.J. Parke, Phys. Rept. 200, 301 (1991)

21. A.D. Martin, M.G. Ryskin, G. Watt, Eur. Phys. J. C 66, 163 (2010)

22. T.D. Coughlin, J.R. Forshaw, JHEP 1001, 121 (2010)

23. M.A. Kimber, A.D. Martin, M.G. Ryskin, Phys. Rev. D 63, 114027 (2001)

24. F.E. Low, Phys. Rev. D 12, 163 (1975) 
25. S. Nussinov, Phys. Rev. Lett. 34, 1280 (1975)

26. M. Diehl, Phys. Rept. 388, 41 (2003)

27. A.G. Shuvaev, K.J. Golec-Biernat, A.D. Martin, M.G. Ryskin, Phys. Rev. D 60, 014015 (1999)

28. A. Shuvaev, Phys. Rev. D 60, 116005 (1999)

29. V.A. Khoze, A.D. Martin, M.G. Ryskin, J. Phys. G 45, 053002 (2018)

30. V.A. Khoze, A.D. Martin, M.G. Ryskin, Eur. Phys. J. C 14, 525 (2000)
31. V.A. Khoze, A.D. Martin, M.G. Ryskin, Eur. Phys. J. C 73, 2503 (2013)

32. L.A. Harland-Lang, A.D. Martin, P. Motylinski, R.S. Thorne, Eur. Phys. J. C 75, 204 (2015)

33. Botje, Comput. Phys. Commun. 182 (2011) 490, arXiv:1005.1481, Erratum arXiv:1602.08383 (2016) 